\title{
Erratum to: The first Italian family with tibial muscular dystrophy caused by a novel titin mutation
}

\author{
Marzia Pollazzon - Tiina Suominen - Sini Penttilä - Alessandro Malandrini • \\ Maria Alessandra Carluccio • Mauro Mondelli • Annabella Marozza • \\ Antonio Federico - Alessandra Renieri - Peter Hackman - Maria Teresa Dotti • \\ Bjarne Udd
}

Published online: 18 December 2009

(C) Springer-Verlag 2009

\section{Erratum to: J Neurol}

DOI 10.1007/s00415-009-5372-3

Under the heading Introduction in line 12, the correct sentence should read: Homozygous mutations more $\mathrm{N}$-terminal of the TMD/LGMD2J mutations and in the C-terminus of TTN cause lethal cardiac and skeletal myopathy [8].
Under the heading Case description in line 11, the correct sentence should read: Electromyogram (EMG) showed myopathic and neurogenic changes only in the muscles of the anterolateral compartment of both legs, without signs in the quadriceps and gastrocnemii.

The online version of the original article can be found under doi:10.1007/s00415-009-5372-3.

M. Pollazzon · A. Marozza · A. Renieri $(\varangle)$

Medical Genetics, Department Molecular Biology,

University of Siena, Policlinico Le Scotte, viale Bracci 2,

53100 Siena, Italy

e-mail: renieri@unisi.it

T. Suominen $\cdot$ S. Penttilä $\cdot$ B. Udd

Neuromuscular Research Center,

University and University Hospital of Tampere,

Tampere, Finland

\author{
A. Malandrini - M. A. Carluccio - A. Federico - M. T. Dotti \\ Section of Neurological Sciences, Department of Neurological, \\ Neurosurgical and Behavioural Sciences, University of Siena, \\ Siena, Italy \\ M. Mondelli \\ EMG Service, ASL7, Siena, Italy \\ P. Hackman · B. Udd \\ Department of Medical Genetics, \\ Folkhälsan Institute of Genetics, \\ University of Helsinki, Helsinki, Finland \\ B. Udd \\ Department of Neurology, Vasa Central Hospital, Vasa, Finland
}

